\title{
Comparison of staining techniques- Ziehl Neelsen stain, Gabbet's Stain, Fluorochrome stain for detection of mycobacterium tuberculosis in sputum
}

\author{
Rupesh Manta ${ }^{1}$, Anila Prabil ${ }^{2, *}$, Prathiba J Dalal ${ }^{3}$ \\ ${ }^{1}$ M.B.B.S Student, ${ }^{2}$ Assistant Professor, ${ }^{3} \mathrm{HOD}$, Dept. of Microbiology, Terna Medical College, Nerul, Navi Mumbai, \\ Maharashtra, India
}

*Corresponding Author:

Email: anilaprabil1@gmail.com

\begin{abstract}
In developing countries, Ziehl-Neelsen (Z-N) sputum smear microscopy is widely used.The present prospective study was under taken to see the efficacy of Ziehl-Neelson method versus Gabbett and Fluorescent staining in the detection of mycobacteria in sputum sample. 80 sputum samples were collected from suspected tuberculosis patients attending RNTCP OPD at, Nerul, Navi Mumbai. Triplicate smears were prepared from all these samples and stained by the Z-N, Gabbet's, and Fluorescent stain. Number of smears positive for Acid Fast Bacilli by, Z-N method 13(16\%), Gabbett's method 7(9\%), Fluorescent method 26(33\%). Combination of two staining methods yielded following results: Z- N + Fluorescent26 (33\%) and Z- N + Gabbett $13(16 \%)$. The conclusion from this study is that when smear examination by $\mathrm{Z}-\mathrm{N}$ is done, there are possibilities of false negative results which can be reduced by using Fluorescent staining. The rate of positivity can be improved by combination of Z-N + Fluorescent staining. So, it may be concluded that combination of $\mathrm{ZN}$ and Fluorescent Microscopy may be considered for diagnosis of pulmonary tuberculosis in any diagnostic set up along with culture which is the gold standard.
\end{abstract}

Keywords: Fluorescent, Gabbett's, Mycobacterium tuberculosis, Revised National Tuberculosis Control Programme (RNTCP), Zehil-Neelson (Z-N).

\section{Introduction}

Pulmonary tuberculosis is a disease of the respiratory system, caused by Mycobacterium tuberculosis. ${ }^{1,2}$ According to World Health Organization (WHO), tubercular infections are currently spreading at the rate of one person per second per million population. ${ }^{3}$ Microscopic examination and culture are essential elements of the bacteriological diagnosis of tuberculosis. The specimen most commonly examined is sputum. ${ }^{2}$ Sputum smear examination is believed to be simple, cheap, quick and practicable and effective case finding method for developing countries. ${ }^{3}$ Since tubercle bacilli are slow growing organisms, and culture results are available only after a period of three to six weeks, an effective microscopy would facilitate the diagnosis and treatment.

Ziehl-Neelsen (Z-N) sputum smear microscopy is a quick but cumbersome procedure because it requires the heat application. ${ }^{4}$ An alternative staining technique is the Gabbet's staining method, where it eliminates the

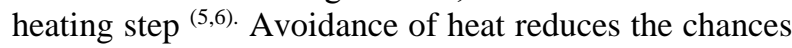
of alteration in physical/chemical composition of the bacteria and there by less chance of alteration in morphology. ${ }^{5,6}$ The Fluorescent staining procedure has been recommended by Revised National Tuberculous Control Programme (RNTCP) where the fluorescent dye (Auramine-O) enters the cell wall of Mycobacterium tuberculosis and makes them glow against dark background using UV light. Microscopic examination under high power objective will reveal mycobacteria as glowing yellow white, rice like bacteria in the smear. When screened using high power objective lens, much larger area can be examined per unit of time. Therefore the present prospective study was under taken to compare Ziehl-Neelson method versus Gabbett and Fluorescent staining in the detection of mycobacteria in sputum samples from patients attending a tertiary care hospital.

\section{Materials and Methods}

80 sputum samples were collected from suspected cases of tuberculosis attending a RNTCP OPD, Terna Medical College, Nerul, Navi Mumbai.

Study Plan: 2months (April-May 2016) at Terna Medical College, Nerul, Navi Mumbai. (Done as a part of Short Term Research Programme for 2 months)

Patients inclusion criteria: Patients attending the RNTCP Centre, having fever, night sweats, cough for more than 3 weeks with sputum, loss of appetite, loss of weight, chest pain, haemoptysis and/ or radiological evidence of tuberculosis were included.

Patient exclusion criteria: Patients previously diagnosed and under treatment.

\section{Sample collected: Sputum}

Three smears were prepared from each sputum sample and stained by the Z-N and Fluorescent staining were done according to the RNTCP guidelines ${ }^{7}$ whereas, Gabbet's staining was done according to Gabbet, H.S. Lancet. ${ }^{1,8}$ All Carbol- fuchsin stained smears were observed under oil immersion and Fluorescent stained smears were examined under high power. 


\section{Results}

In this study, total of 80 sputum samples from suspected tuberculosis patients were examined using different staining methods such as Ziehl-Neelsen method, Gabbett's cold staining method and the Fluorescent staining methods.

Table 1: Results showing number of positive sample using different staining methods

\begin{tabular}{|c|c|c|c|c|c|c|}
\hline $\begin{array}{c}\text { Z-N } \\
\text { Positive } \\
(\mathbf{N}=\mathbf{8 0})\end{array}$ & $\begin{array}{c}\text { Fluorescent } \\
\text { Positive } \\
(\mathbf{N}=\mathbf{8 0})\end{array}$ & $\begin{array}{c}\text { Gabbett's } \\
\text { method } \\
(\mathbf{N}=80)\end{array}$ & $\begin{array}{c}\text { Z.N } \\
\text { Negative } \\
\text { Fluorescent } \\
\text { Positive } \\
(\mathbf{N = 8 0})\end{array}$ & $\begin{array}{c}\text { Z.N } \\
\text { Positive } \\
\text { Fluorescent } \\
\text { Negative }\end{array}$ & $\begin{array}{c}\text { Z.N. } \\
\text { positive } \\
\text { Gabbett } \\
\text { negative } \\
(\mathbf{N = 1 3 )}\end{array}$ & $\begin{array}{c}\text { Z.N. } \\
\text { negative } \\
\text { Gabbett } \\
\text { positive }\end{array}$ \\
\hline $13(16 \%)$ & $26(33 \%)$ & $7(9 \%)$ & $13(16 \%)$ & 0 cases & 6 & 0 cases \\
\hline
\end{tabular}

\section{Discussion}

The present study included 80 samples which were stained and examined by Ziehl-Neelsen method, Gabbett's cold staining method and the Fluorescent staining methods. Of the 80 sputum samples examined, $13(16 \%)$ were positive by Z-N stain, Gabbett's $7(9 \%)$ and Fluorescent 26 (33\%). These results are comparable with the study done by Balakrishna et $\mathrm{al}^{5}$ which shows $18.5,16.5$, and $23.5 \%$ respectively. Similar results are shown by studies done outside India also.

In the conventional Ziehl - Neelsen method, there is better penetration of the stain through the complex cell surface structure due to the heating; therefore the organism appears brighter against a dark background. ${ }^{5}$ In the Z-N staining method, the number of samples positive were $13(16 \%)$ out of the total 80 samples. In spite of being laborious, cost-effectiveness remains the most important advantage of this procedure. ${ }^{5}$

In Gabbett's staining method, only 7(9\%) samples were positive and have failed to detect 6 cases that were $\mathrm{Z}-\mathrm{N}$ positive, which is a major concern and hence it cannot be suggested as microscopy method for diagnosing tuberculosis. This false negativity could be because; the tubercle bacilli appear more delicate and fainter in Gabbett's method than those seen with the Z$\mathrm{N}$ stain. ${ }^{6}$ But the percentage of positivity did not increase even after it was combined i.e, Z-N + Gabbett's 7(9\%).

26(33\%) samples were positive by the Fluorescent stain and when combined Z-N + Fluorescent, the result was $26(33 \%)$ which supports the data of the previous studies. $^{5,3}$ A significant finding was that, thirteen samples which were positive by Fluorescent were negative by Z-N method. This shows that Fluorescent microscopy is a better microscopic method than the conventional Z-N staining. But Fluorescent microscopy is less specific as compared to Z-N method. In the present study it is seen that, combining Z-N +Fluorescent staining increased the positivity rate 26 (33\%) and this data supports the previous study of Soham Gupta et al. ${ }^{5,3}$ Thus, there is significant increase in the rate of positivity on combination of $\mathrm{Z}-\mathrm{N}+$ Fluorescent staining technique than using $\mathrm{ZN}$ alone. So, it may be concluded that Fluorescent Microscopy and Z-N may be used in combination to increase the specificity (Z-N) and sensitivity (Fluorescent). Like in other studies, present study also shows various advantages of fluorescent microscopy, that it enabled the detection of positive smears which were negative with the Ziehl -Neelsen method. ${ }^{8}$ Since these smears are examined under $40 \mathrm{X}^{5,3}$ and also that fluorescing acilli are easily identifiable thus increasing the rate of positivity, reducing the turnaround time and also less eye strain. ${ }^{5,1}$ Previous studies have also shown that, the Fluorescence staining is economical because of its high rate of positivity and less time. ${ }^{9}$ Hence Fluorescent staining has been recently included in Revised National Tuberculosis Control program (RNTCP). ${ }^{7}$ The disadvantage of Fluorescent Microscopy is that it is not cost effective and lacks specificity.

\section{Conclusion}

The conclusion from this study is that when the conventional method of smear examination by $\mathrm{Z}-\mathrm{N}$ is done alone, there are possibilities of false negative results which can be reduced by using Fluorescent staining along with Z-N and the rate of positivity may be improved by combining the conventional Z-N + Fluorescent staining. So, it may be concluded that Fluorescent Microscopy alone may be considered as sufficient microscopic technique for diagnosis of pulmonary tuberculosis in any diagnostic set up along with culture which is the gold standard. From this study it can also be concluded that Gabbett's staining method cannot be used as a standard technique for diagnosing tuberculosis because of its low rate of positivity.

\section{Acknowledgement}

We would like to acknowledge all staff of RNTCP unit for providing with samples.

\section{References}

1. Ba. F Reeder HL-A comparison of FM with the Z.N. technique in the examination of sputum for AFB. Int. J. Tubercle Lung Disease 1999.3(12):1101-5.

2. Deshmukh SR, Mantri SB, Kendre PB and Nagoba BS (1996) A comparison of sputum examination for acid fast bacilli by modified Schaeffer and fulton stain, Ziehl Neelson stain and cold stain, Indian J Med Res.;103;2945 .

3. Roma Goyal et al, IOSR .Journal of Dental and Medical Sciences (IOSR JDMS)Volume 8.Issue 5(July-Aug 2013)PP:05-8. 
4. Soham Gupta et al Journal Brasilerio de Pneumologia.Vol.36 No.5.Sao Paulo Sept/Oct 2010. Manipal, Kasturb a Medical Hospital Pulmonary TB Research Project.

5. Balakrishna et al. Journal of Pharmaceutical Science and Research J. Pharm. Sci and Res. Vol.5 (4)2013:89-92.

6. Gokhale S, Quadir S, Nagar JS, Chakraborty AK. Efficiency of cold staining method of AFB in sputum . A comparison with Ziehl- Neelsen method under field condition. Indin J tuberc. 1990;37:135-7.

7. Operational Research Guidelines and Action Plan Revised National Tuberculosis Control Programme (RNTCP) India, March 2009.

8. Gabbet, H.S. Lancet 1887;1:757.

9. Mustafa Ulukanligil et al. Memorias do Instituto.Oswaldo Cruz Vol.95 N.6.Rio de Janerio. Nov/Dec.2000;855-8.

10. K.Desai et al. Comparative study of Z.N. staining v/s. FS from Pulmonary and Extra Pulmonary TB. Gujarat Medical Journal Aug.09 Vol .64.No.2.

How to cite this article: Manta R, Prabil A, Dalal PJ.

Comparison of staining techniques- Ziehl Neelsen stain, Gabbet's Stain, Fluorochrome stain for detection of mycobacterium tuberculosis in sputum. India. Indian $\mathbf{J}$ Microbiol Res 2018;5(1):44-46. 